\title{
A predictive nomogram of bleeding risk in patients with atrial fibrillation after drug-eluting stent implantation
}

\author{
Jun Qian ${ }^{1}$, Jiyong Zan ${ }^{2}$, Lijun Kuang ${ }^{3}$, Lin Che ${ }^{1}$, Yunan Yu ${ }^{1}$, Ting Shen ${ }^{1}$, Jiani Tang ${ }^{1}$, Fei Chen ${ }^{1}$, \\ Xuebo Liu ${ }^{1}$
}

${ }^{1}$ Department of Cardiology, Tongji Hospital, Tongji University School of Medicine, Shanghai, China; ${ }^{2}$ Department of Gastroenterology, Huazhong University of Science and Technology Union Shenzhen Hospital (Nanshan Hospital), Shenzhen, China; ${ }^{3}$ Department of Ultrasound, Luwan Branch, Ruijin Hospital, Shanghai Jiao Tong University School of Medicine, Shanghai, China

Contributions: (I) Conception and design: J Qian, F Chen, J Zan; (II) Administrative support: F Chen, X Liu, L Che; (III) Provision of study materials or patients: J Qian, F Chen; (IV) Collection and assembly of data: J Qian, L Kuang, Y Yu, T Shen, J Tang; (V) Data analysis and interpretation: J Qian, L Kuang, J Zan, L Che, Y Yu, T Shen, J Tang; (VI) Manuscript writing: All authors; (VII) Final approval of manuscript: All authors.

Correspondence to: Xuebo Liu, MD; Fei Chen, MD. Department of Cardiology, Tongji Hospital, Tongji University School of Medicine, No. 389, Xincun Rd, Putuo District, Shanghai 200065, China. Email: liuxb70@hotmail.com; riverapt@126.com.

\begin{abstract}
Background: The use of anticoagulants and antiplatelet therapies is associated with a higher risk of bleeding in atrial fibrillation (AF) patients after percutaneous coronary intervention, especially after stent implantation. However, no accurate bleeding risk prediction tool has been developed for these patients. The aim of this study was thus to establish a bleeding risk prediction model (predictive nomogram) for patients with AF after stent implantation.
\end{abstract}

Methods: Construction of the predictive nomogram was based on a retrospective study, which enrolled 943 AF patients who underwent drug-eluting stent implantation between May 2012 and September 2016. A range of factors, including demographics, comorbidities, medication strategies, arterial access, and laboratory tests, were collected as baseline data. The least absolute shrinkage and selection operator (LASSO) and multivariate logistic regression analysis were used to identify the key clinical features for construction of the predictive nomogram. The concordance index (C-index) and internal validation were used to evaluate the efficacy of the nomogram.

Results: Of the 943 AF patients that underwent stent implantation, the occurrence of bleeding events was $8.2 \%$ (77 out of 943). Key predictors included the number of antiplatelet drugs, peptic ulcer, cerebral infarction, type 2 diabetes, thrombocytopenia, anemia, prior myocardial infarction, sex (male), use of anticoagulant drugs, liver dysfunction, hypertension, and acute myocardial infarction. These predictors were used to construct the nomogram. The C-index for the prediction of bleeding risk by the nomogram was 0.841 (95\% CI: 0.79-0.89), which indicated good discrimination and calibration. The C-index of internal validation was 0.795 , which demonstrated good efficacy of the model.

Conclusions: This study suggests that our novel nomogram can accurately predict bleeding risk in AF patients after stent implantation during hospitalization, thereby helping to avoid complications. The nomogram may also be helpful for the creation of individualized post-discharge medication strategies.

Keywords: Atrial fibrillation (AF); stent implantation; bleeding risk; nomogram

Submitted May 15, 2020. Accepted for publication Nov 02, 2020.

doi: 10.21037/atm-20-3971

View this article at: http://dx.doi.org/10.21037/atm-20-3971 


\section{Introduction}

Atrial fibrillation (AF) is the most common clinical arrhythmia, and the likelihood of its incidence increases gradually with age. According to the latest management guidelines, AF treatment should be based on an assessment of bleeding and stroke risk (1). Oral anticoagulants are used to prevent thrombosis in AF patients (2), whereas dual antiplatelet therapy (DAPT) is usually recommended for patients undergoing percutaneous coronary intervention (PCI) $(3,4)$. However, an unavoidable practical problem is that approximately one-third of patients with AF also have concomitant coronary heart disease, which requires stent implantation. For AF patients with a high risk of thrombosis after stent implantation, a triple antithrombotic strategy combining oral anticoagulation and DAPT is recommended, although this strategy can result in a higher risk of bleeding compared to dual therapy or DAPT alone (5-7). This combined treatment strategy is still controversial for these patients. Therefore, it is necessary to find novel assessment methods for the early identification of patients at high risk of bleeding.

AF risk factors include electrolyte disturbances, fluid imbalances, neurohormonal disturbances, arrhythmic drugs, and inflammatory reactions (8). Chronic heart failure, hypertension, valvular disease, and myocardial infarction can trigger various common inflammatory pathways, activation of the renin-angiotensin system, and the production of reactive oxygen species, which lead to atrial fibrosis and further promote the occurrence of AF (9). Hypertension, abnormal renal/liver function, stroke, bleeding history or predisposition, labile international normalized ratio (INR), age over 65 years old, drug or alcohol use (HAS-BLED) (10), Anticoagulation and Risk Factors in AF (ATRIA) (11), the modified Outpatient Bleeding Risk Index (mOBRI) (12), and the Reduction of Atherothrombosis for Continued Health (REACH) (13) are factors that have been validated for predicting bleeding risk in AF patients. Nevertheless, none of these factors can accurately predict bleeding risk in AF patients with stent implantation $(14,15)$.

Nomograms are accurate tools for predicting disease or complication risk (16-18). In a recent clinical study on $\mathrm{AF}$, the authors constructed a nomogram model to predict the event-free survival of AF patients before cardiac resynchronization therapy (19). In this study, our predictive nomogram was based on readily available clinical data, and we carried out least absolute shrinkage and selection operator (LASSO) regression analysis to aid in the selection of the best variables. Our aim was to provide a tool for determining personalized post-discharge treatment strategies for patients.

We present the following article in accordance with the STROBE reporting checklist (available at http://dx.doi. org/10.21037/atm-20-3971).

\section{Methods}

\section{Patients}

This retrospective study included AF patients from Shanghai Tongji Hospital between May 2012 and September 2016. The patient inclusion criteria were as follows: aged greater than 18 years old, diagnosis of nonvalvular AF before hospitalization, and drug-eluting stent implantation during hospitalization. The exclusion criteria were as follows: aged less than 18 years old, bioprosthetic or mechanical heart valves, and incomplete clinical information. AF was diagnosed if the standard 12-lead electrocardiogram (ECG) recording or singlelead ECG tracing $\geq 30$ seconds had unrecognized $\mathrm{P}$-wave and irregular R-R interval (indicating atrioventricular conduction was not impaired). Non-valvular AF could be paroxysmal, persistent, or permanent, except for $\mathrm{AF}$ which was secondary to a reversible disease. Coronary angiography and stent implantation were performed via the radial or femoral arteries, and all stents were drug-eluting stents. The choice to use combined antithrombotic therapy after stent implantation was at the discretion of the treating physician. This study was performed in accordance with the Declaration of Helsinki (as revised in 2013). The Shanghai Tongji Hospital Institutional Review Board (IRB) approved the study (approval ID: 2018YYS093), and all patients signed written informed consent before enrollment.

\section{Data collection}

The dataset included demographics, comorbidities, medication strategies, arterial access, and laboratory examinations. Baseline variables were collected from the medical database before stent implantation. Data on anticoagulant drugs, including warfarin and low-molecularweight heparin, were calculated based on whether they were used. Data on antiplatelet drugs, including aspirin and clopidogrel, were calculated by the numbers used by patients. Liver dysfunction was defined as alanine aminotransferase exceeding 3 times the normal limit value. Renal dysfunction 
was defined as an estimated glomerular filtration rate (eGFR) $<30 \mathrm{~mL} / \mathrm{min} / 1.73 \mathrm{~m}^{2}$ or dialysis treatment. Anemia was defined as hemoglobin below $10 \mathrm{~g} / \mathrm{dL}$, and thrombocytopenia was defined as a platelet count below $100,000 / \mu \mathrm{L}$. Bleeding events were defined as any fatal bleeding, including intracranial hemorrhage, intrapericardial hemorrhage, hypovolemic shock/severe hypotension caused by surgical bleeding, a drop in hemoglobin $\geq 3 \mathrm{~g} / \mathrm{dL}$, or an infusion of at least 2 units of red blood cells.

Bleeding events that occurred before discharge were analyzed. Indications for stent implantation included acute coronary syndrome and stable coronary heart disease. Enrolled patients had at least 1 coronary artery lesion in need of a stent. Antiplatelet drugs included aspirin and clopidogrel, and anticoagulant drugs included warfarin and low-molecular-weight heparin.

\section{Statistical analysis}

Statistical analyses were carried out using $\mathrm{R}$ software (version 3.6.1; http://www.R-project.org, R Foundation for Statistical Computing, Vienna, Austria) and the Statistical Package for the Social Sciences (SPSS) 22.0 (SPSS Inc., Armonk, NY, USA). Categorical data are presented as frequencies. Dichotomous variables were compared using the chi-square test or Fisher's exact test. Continuous variables are reported as mean \pm standard deviation. Differences in continuous variables were compared by the Mann-Whitney $U$ test. The optimal variables for predicting bleeding risk in patients with $\mathrm{AF}$ after stent implantation were selected by the LASSO method (20). Non-zero coefficient variables were chosen in the LASSO regression model (21). A prediction model was established using multivariable logistic regression analysis and the combination of the chosen variables. The rms package was used to construct a nomogram based on the logistic regression analysis results. All key predictors were used to construct the predictive nomogram (22), and its efficiency was evaluated using the concordance index (C-index) and a calibration chart. The $\mathrm{C}$-index was used to measure the goodness-of-fit of the model, and a C-index $>0.7$ indicated that the predictive model was a good fit. Internal validation was performed via a calibration chart with 1,000 bootstrapped resamples. The clinical efficacy of the nomogram for predicting bleeding risk was determined by identifying the net benefit under different threshold probabilities and performing decision-curve analysis (23).
Net benefit was calculated by subtracting the proportion of false positives from the proportions of true positives and equilibrating the relative risk of giving up interference against the adverse effects of needless interference (24). The bootstrap method (1,000 resampling) was used for internal verification to avoid potential overfitting, and then a corrected C-index was calculated, which showed that our nomogram model would be useful for predicting bleeding risk. A risk-scoring system was constructed based on the nomogram, and the total scores of all patients were calculated. The bleeding risks of the patients were evaluated according to their different scores.

\section{Results}

\section{Baseline characteristics}

Among 943 patients in the study, bleeding was observed in 77 (8.2\%) cases (bleeding group), while the remaining 866 $(91.8 \%)$ were included in the non-bleeding group. Baseline characteristics, including demographics, comorbidities, medication strategies, arterial access, and laboratory examinations, are listed in Table 1. The average length of hospital stay was 9 days. There were more female and elderly patients in the non-bleeding group than in the bleeding group $(\mathrm{P}<0.001$ and $\mathrm{P}=0.026$, respectively $)$. Moreover, the bleeding group had more complications, including type 2 diabetes, prior cerebral infarction, prior myocardial infarction, liver dysfunction, peptic ulcer, thrombocytopenia, and anemia, compared with the nonbleeding group (all $\mathrm{P}<0.05)$. In addition, more antiplatelet and anticoagulant drugs were used by the bleeding group compared with the non-bleeding group $(\mathrm{P}=0.004$ and $\mathrm{P}=0.032$, respectively). There was no difference in radial and femoral artery access between the two groups $(\mathrm{P}=0.122)$.

\section{Nomogram development and performance}

Among the demographic characteristics, comorbidities, medication strategies, arterial access, and laboratory examinations, 12 key predictors were selected from 16 clinical features with non-zero coefficients in the LASSO regression analysis (Figure 1). These predictors included sex, hypertension, type 2 diabetes, cerebral infarction, acute myocardial infarction, prior myocardial infarction, number of antiplatelet drugs, use of anticoagulants, liver dysfunction, peptic ulcer, thrombocytopenia, and anemia (Table 2). Multivariate logistic regression analysis 
Table 1 Difference between demographic and clinical characteristics of bleeding and non-bleeding groups

\begin{tabular}{|c|c|c|c|}
\hline Characteristics & \multicolumn{2}{|c|}{ Bleeding } & $\mathrm{P}$ \\
\hline Age (years), mean $\pm S D$ & $71.5 \pm 9.7$ & $73.8 \pm 8.5$ & 0.026 \\
\hline Male, n (\%) & $60(77.9)$ & $487(56.2)$ & $<0.001$ \\
\hline Hypertension, n (\%) & $63(81.8)$ & $726(83.8)$ & 0.647 \\
\hline Prior cerebral infarction, n (\%) & $38(49.4)$ & $154(17.8)$ & $<0.001$ \\
\hline Acute MI, n (\%) & $10(13.0)$ & $86(9.9)$ & 0.395 \\
\hline Prior MI, n (\%) & $32(41.6)$ & $159(18.4)$ & $<0.001$ \\
\hline Malignancy, n (\%) & $4(5.2)$ & $39(4.5)$ & 0.780 \\
\hline 1 (aspirin/clopidogrel) & $19(24.7)$ & $292(33.7)$ & \\
\hline 2 (aspirin + clopidogrel) & $55(71.4)$ & $458(52.9)$ & \\
\hline Use of anticoagulant drugs, $n(\%)$ & $21(27.3)$ & $151(17.4)$ & 0.032 \\
\hline Warfarin & $16(20.8)$ & $119(13.7)$ & \\
\hline LMWP & $5(6.5)$ & $32(3.7)$ & \\
\hline Arteries access, $\mathrm{n}(\%)$ & & & 0.122 \\
\hline Radial & $70(90.9)$ & $823(95.0)$ & \\
\hline Femoral & $7(9.1)$ & $43(5.0)$ & \\
\hline
\end{tabular}

MI, myocardial infarction; LMWP, low molecule weight heparin.

revealed that male sex [odds ratio (OR) 2.4341, 95\% confidence interval (CI): 1.2891-4.1990, $\mathrm{P}=0.008]$, type 2 diabetes (OR 3.6463, 95\% CI: 2.0888-6.5702, $\mathrm{P}<0.001$ ), cerebral infarction (OR 4.2944, 95\% CI: 2.4394-7.5794, $\mathrm{P}<0.001)$, prior myocardial infarction (OR 2.9385, $95 \%$ CI: $1.6129-5.3156, \mathrm{P}<0.001)$, number of antiplatelet drugs (OR 3.0221, 95\% CI: 0.8909-14.1893, $\mathrm{P}=0.002$ ), use of anticoagulant drugs (OR 2.2181, 95\% CI: 1.0729-4.5039, $\mathrm{P}=0.029$ ), peptic ulcer (OR 4.5447, 95\% CI: $2.3192-$ 8.7596, $\mathrm{P}<0.001$ ), thrombocytopenia (OR 3.2397, 95\% CI: $1.6708-6.1665, \mathrm{P}<0.001$ ), and anemia (OR 3.1375, 95\% CI: $1.3650-6.8569, \mathrm{P}=0.005)$ were key predictors of bleeding risk in $\mathrm{AF}$ patients after stent implantation. The nomogram was constructed based on the above 12 predictors (Figure 2). Bleeding risk was calculated using the followings steps: First, each variable $\left(2^{\text {nd }}\right.$ to the $13^{\text {th }}$ lines $)$ corresponded to the bleeding score. The bleeding scores of all variables were then summed on the total score axis, and the last line was used to determine bleeding risk (e.g., Total points = 'Sex' score + 'Hypertension' + 'Type 2 diabetes' + 'Cerebral infarction' + 'Acute myocardial infarction' + 'Prior myocardial infarction' + 'Number of antiplatelet drugs' + 'Use of anticoagulant drugs' + ' Liver dysfunction' + 'Peptic ulcer' + 'Thrombocytopenia' + 'Anemia'). In Figure 3, 

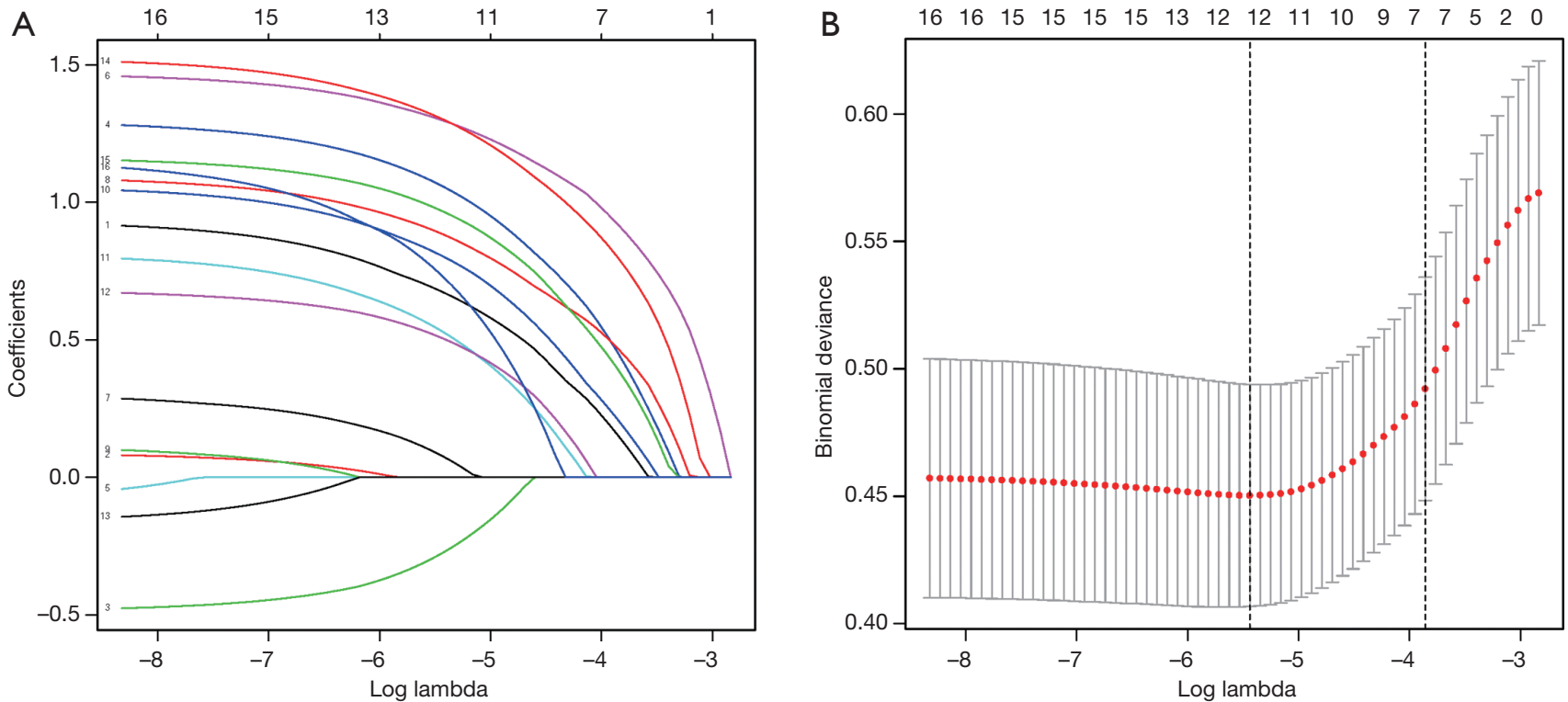

Figure 1 Demographic and clinical feature selection using the LASSO binary logistic regression model. (A) LASSO coefficient profiles of the 16 features. A coefficient profile plot was produced against the log (lambda) sequence. (B) Optimal parameter (lambda) selection in the LASSO model used five-fold cross-validation via minimum criteria. LASSO, least absolute shrinkage and selection operator.

Table 2 Prediction factors for bleeding risk in AF patients after stent implantation

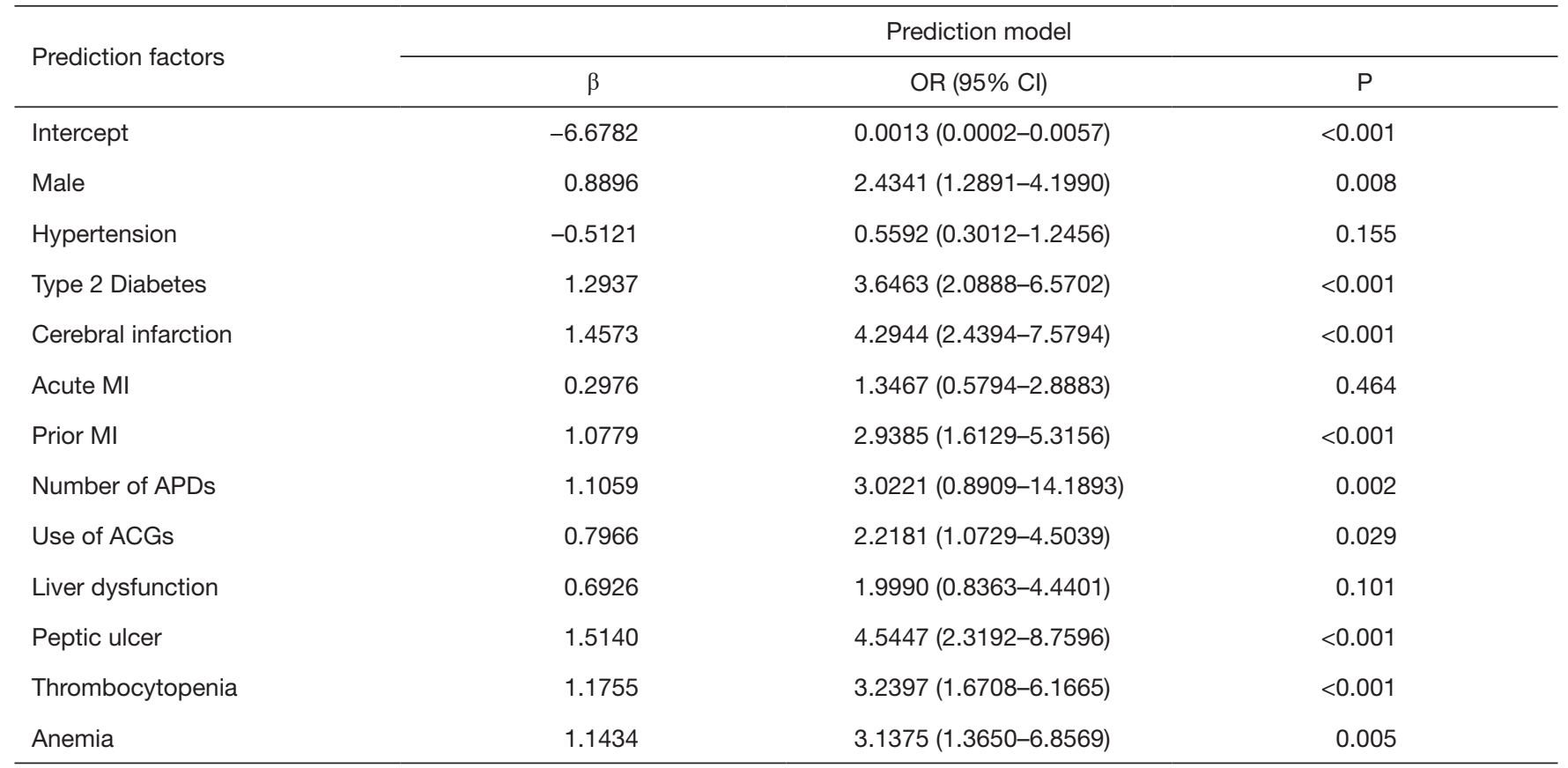

$\beta$ is the regression coefficient. AF, atrial fibrillation; MI, myocardial infarction; APDs, antiplatelet drugs; ACGs, anticoagulant drugs.

the solid line of the nomogram is close to the dotted line on the diagonal, suggesting that the calibration curve has good consistency. The C-index, which is similar to the area under the receiver operating characteristic curve, was used to evaluate the goodness-of-fit of this model. The C-index of this nomogram was 0.841 (95\% CI: 0.791-0.891), which 
Points

Sex

Hypertension

Type 2 diabetes

Cerebral infarction

Acute MI

Prior Ml

Number of antiplatelet drugs

Use anticoagulant drugs

Liver dysfunction

Peptic ulcer

Thrombocytopenia

Anemia

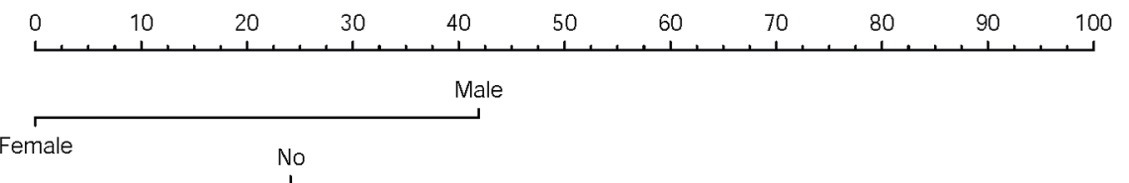

Total Points

Risk of bleeding
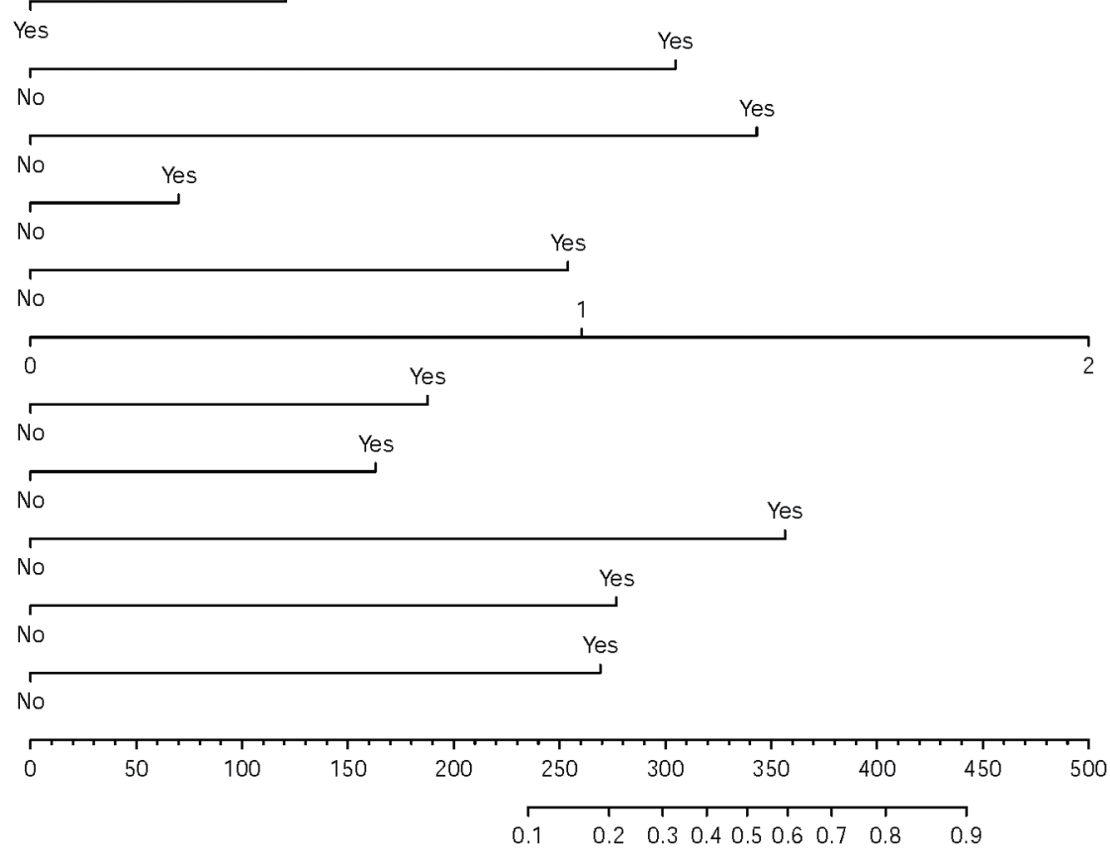

Figure 2 Nomogram of bleeding risk in AF after stent implantation. AF, atrial fibrillation; MI, myocardial infarction; APDs, antiplatelet drugs; ACGs, anticoagulant drugs.

suggested good discrimination, and the C-index of internal validation was 0.795 , which further demonstrated good efficacy of the model. We also constructed a decision curve to guide clinical application of the nomogram (Figure 4). The decision curve indicated that if the threshold probabilities were $>1 \%$ and $<89 \%$, the nomogram could be used to predict bleeding risk. These results verified the high predictive ability of this nomogram.

\section{Discussion}

Based on 12 key predictors screened by LASSO regression, a novel nomogram was constructed that could predict bleeding risk for hospitalized AF patients after stent implantation. Patients with AF may have a high risk of bleeding after stent implantation $(25,26)$, especially when undergoing triple antithrombotic therapy $(27,28)$. Bleeding events occurring after stent implantation are associated with poor prognosis (29). Previous studies have found that patients with medium/high HAS-BLED scores have an increased risk of all-cause death, major adverse cardiovascular events, and major bleeding events (30). HAS-BLED, ATRIA, mOBRI, and REACH are validated bleeding risk-prediction tools. However, their predictive performance in patients with AF receiving triple antithrombotic therapy after stent implantation is unknown.

Overall, the prediction of bleeding risk is complicated, although many clinically important determinants were incorporated into the low $\mathrm{C}$-index in the initial studies $(10,13)$. This nomogram demonstrated that sex (male), type 2 diabetes, cerebral infarction, acute myocardial infarction, prior myocardial infarction, hypertension, a greater number of antiplatelet drugs, use of anticoagulant drugs, liver dysfunction, peptic ulcer, thrombocytopenia, and anemia were the key predictors of bleeding in AF patients after stent implantation. Different access to the radial and femoral arteries did not affect bleeding risk. The selection of the 12 key predictors was based on the LASSO regression model, which is a compressed estimation method used to reduce the variable set (order reduction). By constructing a penalty function, it can compress the coefficients of variables and transform some regression coefficients 


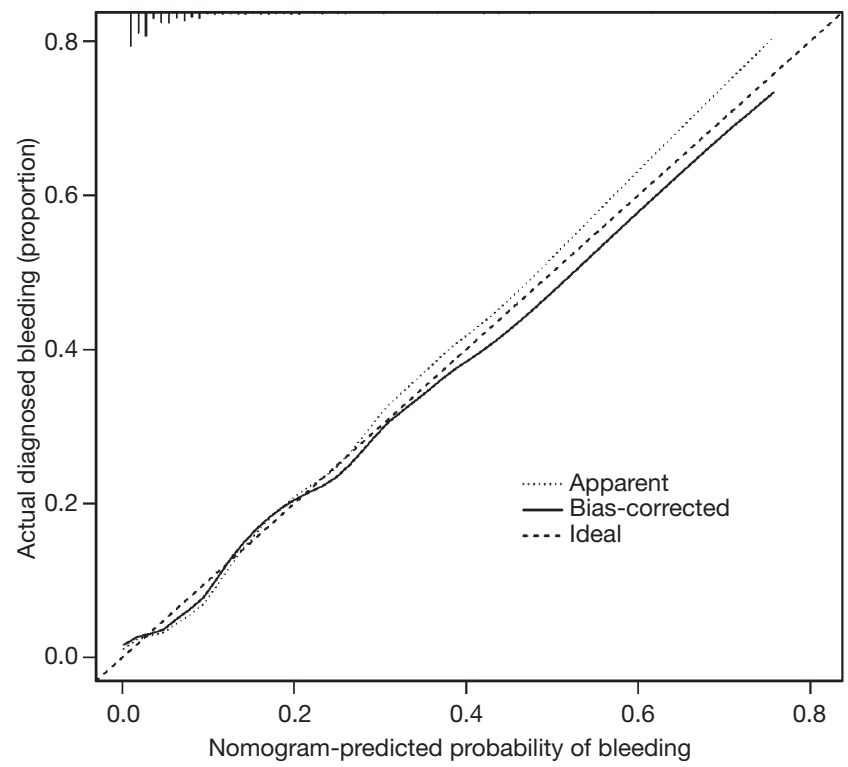

Figure 3 Calibration curves of the nomogram to predict bleeding risk in the population. The $\mathrm{x}$-axis represents the predicted risk of bleeding. The y-axis represents the actual diagnosed bleeding risk. The diagonal dotted line represents a perfect prediction by an ideal model. The solid line represents the performance of the nomogram, where a closer fit to the diagonal dotted line represents better prediction.

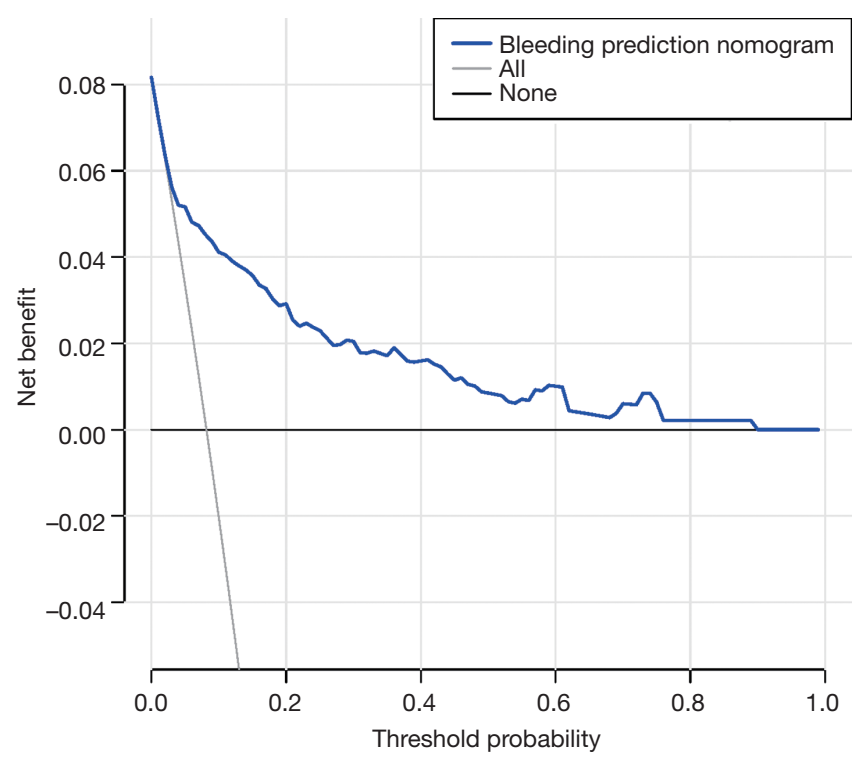

Figure 4 Decision curve analysis for the bleeding risk nomogram. The $y$-axis measures the net benefit. The blue line represents the bleeding risk nomogram. The solid grey line represents the assumption that all patients had bleeding events. The solid black line represents the assumption that no patients had bleeding events. to 0 to achieve the purpose of selecting variables. The recognition of bleeding events is based on the nomogramscoring algorithm. As shown in Figure 2, the corresponding features of each patient are substituted into the nomogram to calculate the score of each feature. Finally, all the scores are added together, and the total score corresponds to the bleeding risk in the last row; thereby, the bleeding risk of the patient can be evaluated.

Previous studies have generally focused on the number of antithrombotic drugs, suggesting that they significantly increase the risk of bleeding in AF patients after stent implantation (31-33), which was further confirmed by this study. An increasing number of studies have also confirmed that the combination of 2 antithrombotic drugs can be more effective in reducing bleeding risk without increasing adverse events (34-36). In this study, we included more clinical factors for better prediction of the bleeding risk in patients. Previous studies have shown that patients with recent or prior myocardial infarction and cerebral infarction have significantly increased bleeding risk $(37,38)$. This is also in agreement with our results and might be related to the increased number of antithrombotic drugs combined with these diseases.

In this study, we also found that sex (male), type 2 diabetes, peptic ulcer, thrombocytopenia, and anemia were independent predictors of bleeding risk, which was consistent with previous research $(3,10,14,38)$. However, in contrast to previous studies $(30,38)$, we identified liver dysfunction, but not renal insufficiency, as a risk factor for bleeding. This could be because only the bleeding events occurring within a few days of stent implantation were counted, whereas liver dysfunction might have a greater impact on acute bleeding events. Overall, this study provides a novel and easy-to-use tool to predict the risk of bleeding more accurately in AF patients after stent implantation, which is of great significance for reducing the incidence of bleeding events in patients. Internal validation in this group indicated that the nomogram had good identification and calibration ability, and the high C-index in internal validation suggests that the nomogram has robust clinical utility.

This study has a few limitations. First, the retrospective design may only represent a small number of AF patients who underwent drug-eluting stent implantation in China. Second, we only validated our results by internal validation but not in a broad external population, which might have led to an overestimation of the nomogram's performance. Third, we counted recent bleeding events that occurred 


\section{Page 8 of 10}

during hospitalization, which might have resulted in bias or missing results.

\section{Conclusions}

Our novel nomogram can be used to predict the bleeding risk of AF patients more accurately after stent implantation during hospitalization. Our data suggests that patients with multiple complications and those using triple antithrombotic drugs have a higher risk of bleeding. By using this model to predict the risk of bleeding in $\mathrm{AF}$ patients, it is possible to reduce the bleeding risk in $\mathrm{AF}$ patients after stent implantation and provide guidance for subsequent medication and treatment strategies.

\section{Acknowledgments}

Funding: This study was supported by the National Natural Science Foundation of China (No. 81670403) and Grant of Shanghai Science and Technology Committee (No. 18411950300, 19XD1403300, and 19411963200).

\section{Footnote}

Reporting Checklist: The authors have completed the STROBE reporting checklist. Available at http://dx.doi. org/10.21037/atm-20-3971

Data Sharing Statement: Available at http://dx.doi. org/10.21037/atm-20-3971

Conflicts of Interest: All authors have completed the ICMJE uniform disclosure form (available at http://dx.doi. org/10.21037/atm-20-3971). The authors have no conflicts of interest to declare.

Ethical Statement: The authors are accountable for all aspects of the work in ensuring that questions related to the accuracy or integrity of any part of the work are appropriately investigated and resolved. This study was in line with the Declaration of Helsinki (as revised in 2013). The Shanghai Tongji Hospital Institutional Review Board (IRB) approved the study (approval ID: 2018YYS093), and all patients signed written informed consent before enrollment.

Open Access Statement: This is an Open Access article distributed in accordance with the Creative Commons
Attribution-NonCommercial-NoDerivs 4.0 International License (CC BY-NC-ND 4.0), which permits the noncommercial replication and distribution of the article with the strict proviso that no changes or edits are made and the original work is properly cited (including links to both the formal publication through the relevant DOI and the license). See: https://creativecommons.org/licenses/by-nc-nd/4.0/.

\section{References}

1. Hindricks G, Potpara T, Dagres N, et al. 2020 ESC Guidelines for the diagnosis and management of atrial fibrillation developed in collaboration with the European Association of Cardio-Thoracic Surgery (EACTS). Eur Heart J 2020. [Epub ahead of print].

2. January CT, Wann LS, Calkins H, et al. 2019 AHA/ ACC/HRS focused update of the 2014 AHA/ACC/HRS guideline for the management of patients with atrial fibrillation: a report of the American college of cardiology/ American heart association task force on clinical practice guidelines and the heart rhythm society. Heart Rhythm 2019;16:e66-93.

3. Baber U, Mehran R, Giustino G, et al. Coronary thrombosis and major bleeding after stent implantation with drug-eluting stents risk scores from Paris. J Am Coll Cardiol 2016;67:2224-34.

4. Dewilde WJM, Oirbans T, Verheugt FWA, et al. Use of clopidogrel with or without aspirin in patients taking oral anticoagulant therapy and undergoing percutaneous coronary intervention: an open-label, randomised, controlled trial. Lancet 2013;381:1107-15.

5. Yasuda S, Kaikita K, Akao M, et al. Antithrombotic therapy for atrial fibrillation with stable coronary disease. N Engl J Med 2019;381:1103-13.

6. Cannon CP, Bhatt DL, Oldgren J, et al. Dual antithrombotic therapy with dabigatran after stent implantation in atrial fibrillation. $\mathrm{N}$ Engl J Med 2017;377:1513-24.

7. Golwala HB, Cannon CP, Steg PG, et al. Safety and efficacy of dual vs. Triple antithrombotic therapy in patients with atrial fibrillation following percutaneous coronary intervention: a systematic review and metaanalysis of randomized clinical trials. Eur Heart J 2018;39:1726.

8. Bosch NA, Cimini J, Walkey AJ. Atrial Fibrillation in the ICU. Chest 2018;154:1424-34.

9. Aldhoon B, Melenovský V, Peichl P, et al. New insights into mechanisms of atrial fibrillation. Physiol Res 
2010;59:1-12.

10. Lip GY, Frison L, Halperin JL, et al. Comparative validation of a novel risk score for predicting bleeding risk in anticoagulated patients with atrial fibrillation: the hasbled (hypertension, abnormal renal/liver function, stroke, bleeding history or predisposition, labile inr, elderly, drugs/alcohol concomitantly) score. J Am Coll Cardiol 2011;57:173-80.

11. Fang MC, Go AS, Chang Y, et al. A new risk scheme to predict warfarin-associated hemorrhage: the atria (anticoagulation and risk factors in atrial fibrillation) study. J Am Coll Cardiol 2011;58:395-401.

12. Airaksinen KE, Suurmunne H, Porela P, et al. Usefulness of outpatient bleeding risk index to predict bleeding complications in patients with long-term oral anticoagulation undergoing coronary stenting. Am J Cardiol 2010;106:175-9.

13. Ducrocq G, Wallace JS, Baron G, et al. Risk score to predict serious bleeding in stable outpatients with or at risk of atherothrombosis. Eur Heart J 2010;31:1257-65.

14. Kiviniemi T, Puurunen M, Schlitt A, et al. Performance of bleeding risk-prediction scores in patients with atrial fibrillation undergoing percutaneous coronary intervention. Am J Cardiol 2014;113:1995-2001.

15. Puurunen MK, Kiviniemi T, Schlitt A, et al. Chads2, cha2ds2-vasc and has-bled as predictors of outcome in patients with atrial fibrillation undergoing percutaneous coronary intervention. Thromb Res 2014;133:560-6.

16. Wang Y, Li J, Xia Y, et al. Prognostic nomogram for intrahepatic cholangiocarcinoma after partial hepatectomy. J Clin Oncol 2013;31:1188-95.

17. Zhong H, Chen J, Cheng S, et al. Prognostic nomogram incorporating inflammatory cytokines for overall survival in patients with aggressive non-hodgkin's lymphoma. EBioMedicine 2019;41:167-74.

18. Kumbhani DJ, Wells BJ, Lincoff AM, et al. Predictive models for short- and long-term adverse outcomes following discharge in a contemporary population with acute coronary syndromes. Am J Cardiovasc Dis 2013;3:39-52.

19. Cai M, Hua $W$, Zhang N, et al. A prognostic nomogram for event-free survival in patients with atrial fibrillation before cardiac resynchronization therapy. BMC Cardiovasc Disord 2020;20:221.

20. Sauerbrei W, Royston P, Binder H. Selection of important variables and determination of functional form for continuous predictors in multivariable model building. Stat Med 2007;26:5512-28.
21. Kidd AC, McGettrick M, Tsim S, et al. Survival prediction in mesothelioma using a scalable lasso regression model. instructions for use and initial performance using clinical predictors. BMJ Open Respir Res 2018;5:e000240.

22. Balachandran VP, Gonen M, Smith JJ, et al. Nomograms in oncology: more than meets the eye. Lancet Oncol 2015;16:e173-80.

23. Vickers AJ, Cronin AM, Elkin EB, et al. Extensions to decision curve analysis, a novel method for evaluating diagnostic tests, prediction models and molecular markers. BMC Med Inform Decis Mak 2008;8:53.

24. Huang YQ, Liang CH, He L, et al. Development and validation of a radiomics nomogram for preoperative prediction of lymph node metastasis in colorectal cancer. J Clin Oncol 2016;34:2157-64.

25. Lopes RD, Heizer G, Aronson R, et al. Antithrombotic therapy after acute coronary syndrome or stent implantation in atrial fibrillation. N Engl J Med 2019;380:1509-24.

26. Gibson CM, Mehran R, Bode C, et al. Prevention of bleeding in patients with atrial fibrillation undergoing stent implantation. N Engl J Med 2016;375:2423-34.

27. Moser M, Olivier CB, Bode C. Triple antithrombotic therapy in cardiac patients: more questions than answers. Eur Heart J 2014;35:216-23.

28. Lamberts M, Olesen JB, Ruwald MH, et al. Bleeding after initiation of multiple antithrombotic drugs, including triple therapy, in atrial fibrillation patients following myocardial infarction and coronary intervention: a nationwide cohort study. Circulation 2012;126:1185-93.

29. Chhatriwalla AK, Amin AP, Kennedy KF, et al. Association between bleeding events and in-hospital mortality after percutaneous coronary intervention. JAMA 2013;309:1022-29.

30. Ruiz-Nodar JM, Marin F, Roldan V, et al. Should we recommend oral anticoagulation therapy in patients with atrial fibrillation undergoing coronary artery stenting with a high has-bled bleeding risk score? Circ Cardiovasc Interv 2012;5:459-66.

31. Capodanno D, Huber K, Mehran R, et al. Management of antithrombotic therapy in atrial fibrillation patients undergoing stent implantation: jacc state-of-the-art review. J Am Coll Cardiol 2019;74:83-99.

32. Lopes RD, Hong H, Harskamp RE, et al. Safety and efficacy of antithrombotic strategies in patients with atrial fibrillation undergoing percutaneous coronary intervention: a network meta-analysis of randomized controlled trials. JAMA Cardiol 2019;4:747-55. 


\section{Page 10 of 10}

33. Haller PM, Sulzgruber P, Kaufmann C, et al. Bleeding and ischaemic outcomes in patients treated with dual or triple antithrombotic therapy: systematic review and meta-analysis. Eur Heart J Cardiovasc Pharmacother 2019;5:226-36.

34. Vora AN, Alexander JH, Wojdyla D, et al. Hospitalization among patients with atrial fibrillation and a recent acute coronary syndrome or percutaneous coronary intervention treated with apixaban or aspirin: insights from the Augustus trial. Circulation 2019;140:1960-3.

35. Sindet-Pedersen C, Lamberts M, Staerk L, et al. Combining oral anticoagulants with platelet inhibitors in patients with atrial fibrillation and coronary disease. J Am Coll Cardiol 2018;72:1790-800.

Cite this article as: Qian J, Zan J, Kuang L, Che L, Yu Y, Shen T, Tang J, Chen F, Liu X. A predictive nomogram of bleeding risk in patients with atrial fibrillation after drugeluting stent implantation. Ann Transl Med 2021;9(3):193. doi: 10.21037/atm-20-3971

\section{Qian et al. Prediction of bleeding risk in AF after stent implantation}

36. Bainey KR, Morais J, Zeymer U, et al. Atrial fibrillation with percutaneous coronary intervention: navigating the minefield of antithrombotic therapies. Atherosclerosis 2019;289:118-25.

37. Sutton NR, Seth M, Ruwende C, et al. Outcomes of patients with atrial fibrillation undergoing percutaneous coronary intervention. J Am Coll Cardiol 2016;68:895-904.

38. Natsuaki M, Morimoto T, Yamaji K, et al. Prediction of thrombotic and bleeding events after percutaneous coronary intervention: CREDO-Kyoto thrombotic and bleeding risk scores. J Am Heart Assoc 2018;7:e008708.

(English Language Editor: C. Betlazar-Maseh; Quality Control Editor: J. Gray) 